\title{
A new time-hopping/direct-sequence biorthogonal PPM UWB communication system
}

\author{
Ye-Shun Shen ${ }^{1}$, Fang-Biau Ueng ${ }^{2}$ and Li-Der Jeng ${ }^{3^{*}}$
}

\begin{abstract}
In order to increase the capacity and diminish the multiple access interference (MAI) of an ultra-wideband (UWB) system, we propose a new time-hopping/direct-sequence (TH/DS) scheme using $\mathrm{N}$-ary biorthogonal pulse position modulation (BPPM). In contrast with the conventional TH/DS systems employing fixed partition of the TH slots (Shen and Ueng, Proceedings of the IEEE VTC-Spring, 2010), the proposed TH/DS system can put the start location of the TH slot in any one of $Q$ available pulse positions within the frame. In the proposed TH/DS system, the modulation level of BPPM can be increased and the multiple access capability can be improved without degrading the system performance. Compared with the existing TH-UWB system that employs the whole frame to carry out TH process (Shen et al. IEEE Trans. Veh. Technol. 59(2), 742-753, 2010), the proposed system has the merits of smooth power spectral density and low receiver complexity. In this article, we also derive the probability distribution of MAI for each correlator's output of the proposed TH/DS system based on the analytic characteristic function technique. In order to verify the correctness of the performance analyses and to demonstrate the effectiveness of the proposed TH/DS system, some simulation results are given in both the additive white Gaussian noise channel and the realistic UWB fading channels. From the simulation results, we find that the proposed TH/DS system outperforms the conventional TH/DS scheme.
\end{abstract}

Keywords: characteristic function (CF), time-hopping (TH), ultra-wideband (UWB), multiple access interference (MAl)

\section{Introduction}

Owing to the demand of short-range high-speed wireless data communication, the impulse radio ultra-wideband (IR-UWB) transmission which transmits extremely short impulses (referred to as monocycles) becomes an attractive technology recently [1]. The high ratio of transmitted signal bandwidth to data signal bandwidth makes UWB technique useful for multiple access applications. The modulation schemes that consist of pulse amplitude modulation (PAM), pulse position modulation (PPM), and pulse position amplitude modulation (PPAM) are widely adopted in IR-UWB systems. PPM and PAM modulations use the precise position and amplitude of impulses, respectively, to convey data message, while PPAM exploits both the position and amplitude of mono-cycle to carry information. $N(=2 M)$-ary biorthogonal PPM (BPPM) which combines binary PAM and

\footnotetext{
* Correspondence: lider@cycu.edu.tw

${ }^{3}$ Department of Electronic Engineering, Chung-Yuan Christian University,

Chung Li, Taiwan

Full list of author information is available at the end of the article
}

$M$-ary PPM is a special case of PPAM. Under the same throughput, $N$-ary BPPM can provide better performance and less complexity than those of $M$-ary PPM $[2,3]$.

The time-hopping (TH) and direct sequence (DS) multiple access schemes are applied in IR-UWB systems. In the conventional TH UWB system, each symbol duration is divided into $N_{s}$ frames, and each frame interval is divided into $N_{c}$ time slots (chips). A data symbol is modulated based on the adopted modulation scheme to transmit one pulse in each frame duration. Afterward, the position of the time slot in each frame on which the modulated pulse is located is selected and hopped from frame to frame according to the pseudorandom $\mathrm{TH}$ code. However, the use of PPM and/or PAM signaling in conventional $\mathrm{TH}$ system has one disadvantage that the line spectral occurs in the spectrum of the transmitted signals. This is because the same polarity (unipolar) of monocycles are transmitted in a given symbol period. To alleviate the effect of this problem, the conventional $\mathrm{TH}$ systems exploiting the randomized polarity
(C) 2011 Shen et al; licensee Springer. This is an Open Access article distributed under the terms of the Creative Commons Attribution License (http://creativecommons.org/licenses/by/2.0), which permits unrestricted use, distribution, and reproduction in any medium, provided the original work is properly cited. 
(bipolar) of the transmitted pulses, also called the hybrid TH/DS system, are studied in [3,4]. The selections of the user-specific TH codes, corresponding to the utilized time slots, and the polarity (DS) codes are well designed to mitigate the multiple access interference (MAI) and improve the system capability.

In Figure 1, all of the users transmit their signals in the same symbol period. The matrix representations of

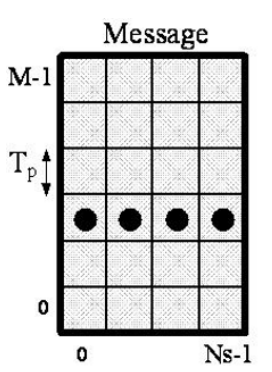

Desired user:

Multiple-Access Interference:

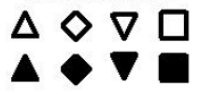

Data symbols:

$\boldsymbol{m}^{(1)}=2, \boldsymbol{n}^{(1)}=1$

$\boldsymbol{m}^{(2)}=5, \boldsymbol{n}^{(2)}=0$

$\boldsymbol{m}^{(3)}=1, \boldsymbol{n}^{(3)}=1$

$\boldsymbol{m}^{(4)}=0, \boldsymbol{n}^{(3)}=1$

$\boldsymbol{m}^{(5)}=4, \boldsymbol{n}^{(5)}=0$

$\mathrm{T}_{\mathrm{f}}$
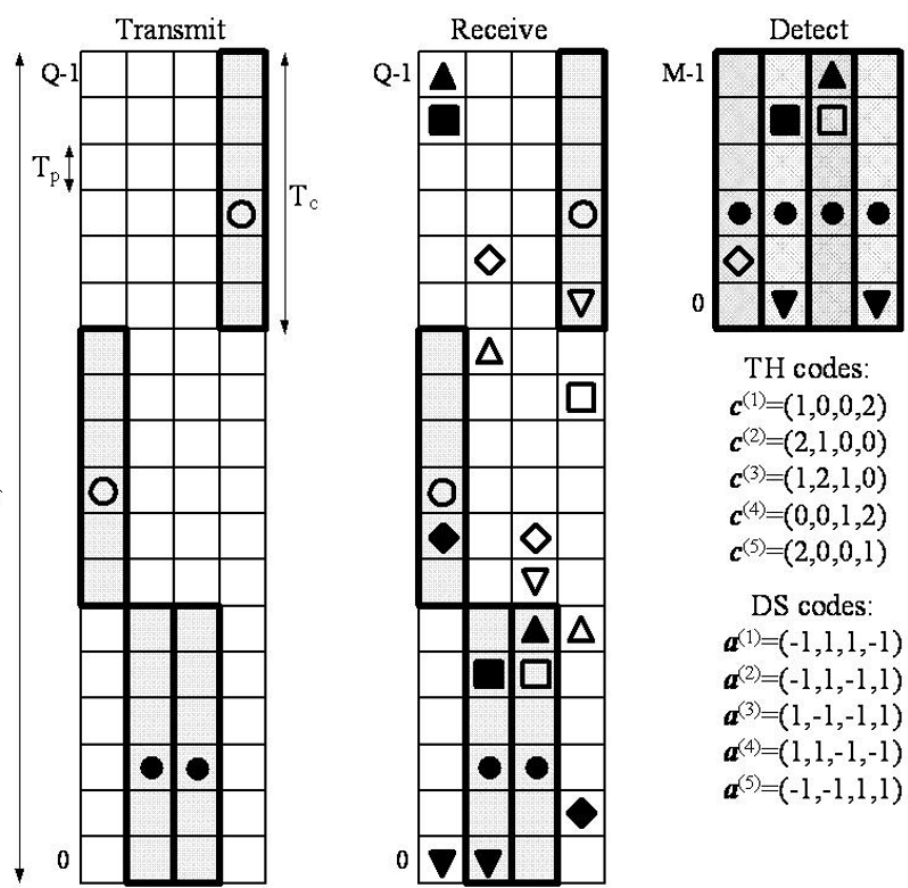

TH codes:

$\boldsymbol{c}^{(1)}=(1,0,0,2)$

$\boldsymbol{c}^{(2)}=(2,1,0,0)$

$\boldsymbol{c}^{(3)}=(1,2,1,0)$

$\boldsymbol{c}^{(4)}=(0,0,1,2)$

$\boldsymbol{c}^{(5)}=(2,0,0,1)$

DS codes:

$\boldsymbol{a}^{(1)}=(-1,1,1,-1)$

$\boldsymbol{a}^{(2)}=(-1,1,-1,1)$

$\boldsymbol{a}^{(3)}=(1,-1,-1,1)$

$\boldsymbol{a}^{(4)}=(1,1,-1,-1)$

$\boldsymbol{a}^{(5)}=(-1,-1,1,1)$

(a)

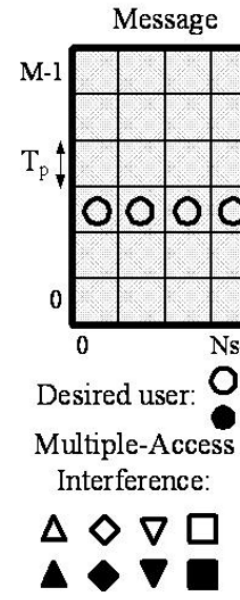

Data symbols: $\boldsymbol{m}^{(1)}=\mathbf{2}, \boldsymbol{n}^{(1)}=\mathbf{0}$ $\boldsymbol{m}^{(2)}=5, \boldsymbol{n}^{(2)}=1$ $\boldsymbol{m}^{(3)}=1, \boldsymbol{n}^{(3)}=1$

$\boldsymbol{m}^{(4)}=0, \boldsymbol{n}^{(4)}=0$ $\boldsymbol{m}^{(5)}=4, \boldsymbol{n}^{(5)}=0$

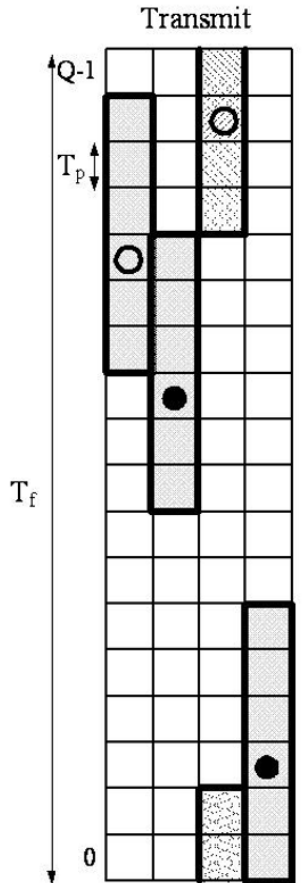

(b)
Detect

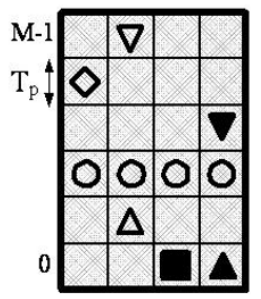

TH codes: $\boldsymbol{c}^{(1)}=(11,8,14,0)$ $\boldsymbol{c}^{(2)}=(15,4,0,13)$ $\boldsymbol{c}^{(3)}=(14,3,7,12)$ $\boldsymbol{c}^{(4)}=(8,13,4,3)$

$\boldsymbol{c}^{(5)}=(1,16,10,5)$

DS codes: $\boldsymbol{a}^{(1)}=(1,-1,1,-1)$ $\boldsymbol{a}^{(2)}=(1,1,-1,-1)$ $\boldsymbol{a}^{(3)}=(-1,1,1,-1)$ $\boldsymbol{a}^{(4)}=(-1,-1,1,1)$ $\boldsymbol{a}^{(5)}=(-1,1,-1,1)$

Figure 1 The matrix representations of hopping and dehopping processes for (a) the conventional TH/DS and (b) the proposed TH/DS systems. 
the $\mathrm{TH}$ and dehopping processes are carried out in a deterministic manner. A drawback of the conventional $\mathrm{TH}$ system is that the greater the modulation level of the $N$-ary BPPM or the number of frame $\left(\widehat{N}_{s}\right)$ is, the fewer the number of the provided TH slots $\left(\widehat{N}_{c}\right)$ becomes. In this article, a flexible $\mathrm{TH}$ scheme that employs the whole frame duration to carry out $\mathrm{TH}$ is proposed. In the proposed system, each symbol duration is divided into $N_{s}$ frames, but each frame interval is partitioned into $Q$ pulse slots. Specifically, each user is assigned a random $\mathrm{TH}$ code such that the first pulse position of a TH slot can be located to any one of $Q$ available pulse slots within one frame. Therefore, the MAI in the proposed system is hardly produced from the same interfering user's signal because of the randomness location of the transmitted pulses within each used TH slot. However, in the conventional TH scheme, the MAI that leads to an erroneous symbol detection is often produced from the same interfering user's signal. When the number of frame is increased to be the largest, then the number of TH slot becomes $\widehat{N}_{c}=1$, i.e., there is no $\mathrm{TH}$ capability to be provided in the conventional system. In the same scenario, the TH capability still exists in the proposed system. Therefore, the impact of MAI on the system performance in the conventional scheme is more severe than that in the proposed scheme. Hence, irrespective of the modulation level of BPPM, the performances of the proposed scheme is always better than that of the conventional scheme.

In asynchronous MA environment, the collisions of the received pulses from different users are inevitable because of the randomness of time misalignment among the received signals of all the users. Compared with binary modulation, it is well known that $N$-ary biorthogonal modulations are able to provide higher throughput and better BER performance, as the modulation level is increased [5]. The benefit of the proposed TH scheme is able to increase the modulation level of $\mathrm{N}$-ary BPPM signaling without decreasing the number of TH slots. Consequently, the proposed $\mathrm{TH}$ method employing larger modulation level can carry more information bits in a symbol duration and then improve the system throughput. At the transmitter of the proposed TH/DS system, the TH-coded symbol sequence of each user is first generated according to the specific TH code (hopping pattern) and becomes as the input of $N$-ary BPPM modulator which also applies specific DS code to randomize the polarity of the modulated pulses. The proposed $\mathrm{TH}$ technique has been widely employed in the frequency-hopping $(\mathrm{FH})$ system which combines a larger modulation level of $M$-ary FSK modulation and provides better performance [6,7].
For the conventional TH-UWB systems with binary PAM and/or PPM modulations in asynchronous MA scenarios, the performance analyses have been extensively investigated in $[4,8-15]$. For the conventional THUWB systems with $M$-ary orthogonal PPM and $N$-ary biorthogonal PPM modulations, some relative studies have been reported in [16-20] and [2,21]. The Gaussian distribution assumption can be adopted to model the MAI statistics to derive some simple theoretical analyses for the binary PPM, $M$-ary orthogonal PPM and $N$-ary biorthogonal PPM signaling $[2,8-10,16]$. However, if we consider the medium and high signal-to-noise ratio (SNR) conditions, the Gaussian approximation (GA) fails to model the statistics of MAI precisely $[4,11-13,18,20]$. The exact expression of the cumulative distribution function (CDF) of the MAI is inconsistent with that obtained by using the GA. Hence, using GA leads to inaccurate error probability analysis and also leads to optimistically overestimate the system performance. By deriving the characteristic functions (CF) of the MAI, the accurate performance analyses of binary $\mathrm{PPM} / \mathrm{TH}, M$-ary orthogonal PPM/TH and $N$-ary BPPM/ TH/DS UWB systems were proposed in [3,12-14,20]. In this article, the analytic CF expression of the MAI is derived and the performance analyses of the proposed TH/DS-UWB system is then obtained.

The rest of this article is organized as follows. The conventional and the proposed TH/DS systems with $N$ ary BPPM are described in Section 2. In Section 3, the analytic expression of the probability distributions of MAI and the average symbol error rate (SER) of the proposed TH/DS system is derived. Some numerical examples and discussions for the proposed system are presented in Section 4. Finally, we give some conclusions in Section 5.

\section{System model}

In this section, the performance of the proposed $\mathrm{TH} /$ DS-UWB system will be compared with that of the conventional DS-UWB system and the conventional TH/DS UWB system. The conventional DS-UWB signal can be expressed as follows $[13,14]$ :

$$
s_{\mathrm{DS}}^{(k)}(t)=\sqrt{\frac{E_{\mathrm{b}}}{N_{r}}} \sum_{j=-\infty}^{\infty} \sum_{n=0}^{N_{r}-1} d_{j}^{(k)} c_{n}^{(k)} p\left(t-j T_{f}-n T_{c}\right)
$$

where $c_{n}^{(k)} \in\{1,-1\}$ denotes the $n$th chip of the $k$ th user's spreading sequence; $E_{\mathrm{b}}$ is the average bit energy; $d_{j}^{(k)} \in\{1,-1\}$ is the $j$ th message bit of the $k$ th user; and $N_{r}$ denotes the number of chips in a bit duration. For the conventional TH/DS system using $N$-ary BPPM, we divide one symbol duration $T_{s}$ into $N_{s}$ equally spaced 
frames with duration $T_{f}$, and each frame interval $T_{f}$ is divided into $N_{c}$ equally spaced time slots (chips) with duration $T_{c}$. The $N$-ary BPPM-modulated signal is transmitted and located on one of the available $N_{c}$ chips according to the assigned $\mathrm{TH}$ code for each user. The $\mathrm{N}$-ary BPPM-modulated signal is the antipodal version of the $M(=N / 2)$-ary PPM signal. For the $i$ th symbol period of the $k$ th user, the $N$-ary BPPM signal of the conventional TH/DS system can be modeled as

$$
s^{(k)}(t)=\sum_{i}(-1)^{n_{i}^{(k)}} b^{(k)}\left(t-i T_{s}-m_{i}^{(k)} \delta\right)
$$

where $t$ is the time index, $m_{i}^{(k)} \in\{0,1,2, \ldots, M-1\}$ and $n_{i}^{(k)} \in\{0,1\}$ denote $v-1$ bits and one bit of the $i$ th message symbol, respectively. Overall, $u_{i}^{(k)}=\left(m_{i}^{(k)}, n_{i}^{(k)}\right)$ represents a $v$-bit message symbol and maintains the same in the $i$ th symbol duration. In addition, the signal waveform of the $k$ th user can be written as

$$
b^{(k)}(t)=\sqrt{\frac{E_{s}}{N_{s}}} \sum_{j=i N_{s}}^{(i+1) N_{s}-1} a_{j}^{(k)} p\left(t-j T_{f}-c_{j}^{(k)} T_{c}\right)
$$

where $E_{\mathrm{s}}$ is the average symbol energy which is assumed to be the same for all the users' signals, $N_{s}$ is the number of transmitted pulses required to represent one symbol of message, $c_{j}^{(k)} \in\left\{0,1,2, \ldots, N_{c}-1\right\}$ is the $j$ th element of the $k$ th user's TH code, and $a_{j}^{(k)} \in\{-1,1\}$ is the $j$ th element of the $k$ th user's random polarity code (or random DS spreading code). To reduce the effect of MAI, it can be well designed to assign the specific random $\mathrm{TH}$ code $\mathbf{c}^{(k)}=\left(c_{0}^{(k)}, c_{1}^{(k)}, \ldots, c_{N_{s}-1}^{(k)}\right)$ and random polarity code $\mathbf{a}^{(k)}=\left(a_{0}^{(k)}, a_{1}^{(k)}, \ldots a_{N_{s}-1}^{(k)}\right)$ to the $k$ th user. $\delta$ denotes the time shift between two adjacent positions for the BPPM signals and is selected to be the pulse width $T_{p}$ due to the assumption of orthogonal BPPM signaling. Therefore, the chip duration on which a $M$ ary PPM signal is located is equal to $T_{c}=M T_{p}$. It is worthy to note that each frame duration is partitioned into $N_{c}$ non-overlapped time (chip) slots, the pulse positions of the $N_{s}$ transmitted BPPM signals in the $N_{s}$ selected chip slots are the same and are illustrated in Figure 1a.

For UWB systems, several pulse waveforms have been proposed. The normalized second-order Gaussian monocycle, $p(t)=\left[1-4 \pi\left(t / \tau_{p}\right)^{2}\right] \exp \left[-2 \pi\left(t / \tau_{p}\right)^{2}\right]$, which has been widely applied in many studies of the literature is adopted in this article as data bearing waveform. The duration of the normalized second-order Gaussian monocycle is $T_{p}$. In addition, the normalized autocorrelation function of $p(t)$ is defined as $\int_{-\infty}^{\infty} p(t) p(t-\tau) d t=\left[1-4 \pi\left(\tau / \tau_{p}\right)^{2}+4 \pi^{2} / 3 \cdot\left(\tau / \tau_{p}\right)^{4}\right] \exp \left[-\pi\left(\tau / \tau_{p}\right)^{2}\right][8,12]$.

The block diagram of the proposed TH/DS-UWB system with $N$-ary BPPM signaling is depicted in Figure 2. The data bit stream is with rate $R_{b}$ bits/s and is then mapped into an $N$-ary BPPM symbol sequence with rate $R_{s}$ symbols/s, where $R_{s}=1 / T_{s}=1 / v T_{b}$, and $v=\log _{2} N$ is the number of bits per symbol. In the proposed system, each frame interval is partitioned into $Q$ equally spaced pulse slots with duration $T_{p}$. The user's signal can be located in the whole frame duration, i.e., $Q$ overlapped and cyclic TH slots shown in Figure 1b, to carry out $\mathrm{TH}$ process. In the proposed TH/DS system, the number of utilized pulse positions for $N$-ary BPPM signaling, i.e., $M=N / 2$, can be chosen as $M \leq Q$. For the conventional TH/DS system, the matrix representations of $\mathrm{TH}$ and dehopping processes are illustrated in Figure 1a. In the proposed TH/DS system (Figure 1b), the $k$ th user is assigned a specific TH address code $\mathbf{c}^{(k)}=\left(c_{0}^{(k)}, c_{1}^{(k)}, \ldots, c_{N_{s}-1}^{(k)}\right)$ and a random polarity (DS) code $\mathbf{a}^{(k)}=\left(a_{0}^{(k)}, a_{1}^{(k)}, \ldots a_{N_{s}-1}^{(k)}\right)$, where $c_{j}^{(k)} \in G=\{0,1,2, \ldots, Q-1\} . c_{j}^{(k)}$ denotes the $j$ th code element of the TH code to locate the first pulse position of the $N$-ary BPPM signaling in the $j$ th frame duration. $a_{j}^{(k)} \in\{-1,1\}$ is the $j$ th element of the DS code. The signal of the proposed TH/DS system with $N$-ary BPPM signal is given by

$$
s_{\text {new }}^{(k)}(t, i)=\sqrt{\frac{E_{s}}{N_{s}}} \sum_{i=-\infty}^{\infty} \sum_{j=i N_{s}}^{(i+1) N_{s}-1}(-1)^{n_{i}^{(k)}} a_{j}^{(k)} p\left(t-j T_{f}-b_{j}^{(k)} \delta\right)
$$

where $N_{s}$ is the number of frames. $N_{s}$ pulses are transmitted to represent a $v$-bit message symbol $u_{i}^{(k)}=\left(m_{i}^{(k)}, n_{i}^{(k)}\right)$. $E_{s}$ denotes the energy of a monocycle. $b_{j}^{(k)}$ can be obtained as $b_{j}^{(k)}=c_{j}^{(k)} \oplus m_{i}^{(k)}$, $m_{i}^{(k)} \in\{0,1,2, \ldots, M-1\}$ denotes a $(v-1)$-bit data symbol and $\oplus$ denotes the modulo- $Q$ addition. Specifically, the values of the two symbols, $b_{j}^{(k)}$ and $a_{j}^{(k)}$, determine, respectively, the position and amplitude of a $N$ ary BPPM signal transmitted in the $j$ th frame duration. The complexities of the conventional and the proposed TH/DS schemes are almost the same if small $N(8,16$, or 32) is adopted. However, the proposed TH/DS system can adopt large $N(64,128$, or 256) that is infeasible for the conventional TH/DS system. In other words, the proposed TH/DS scheme is more flexible than the conventional TH/DS scheme. In asynchronous multipath additive white Gaussian noise (AWGN) channel, the 


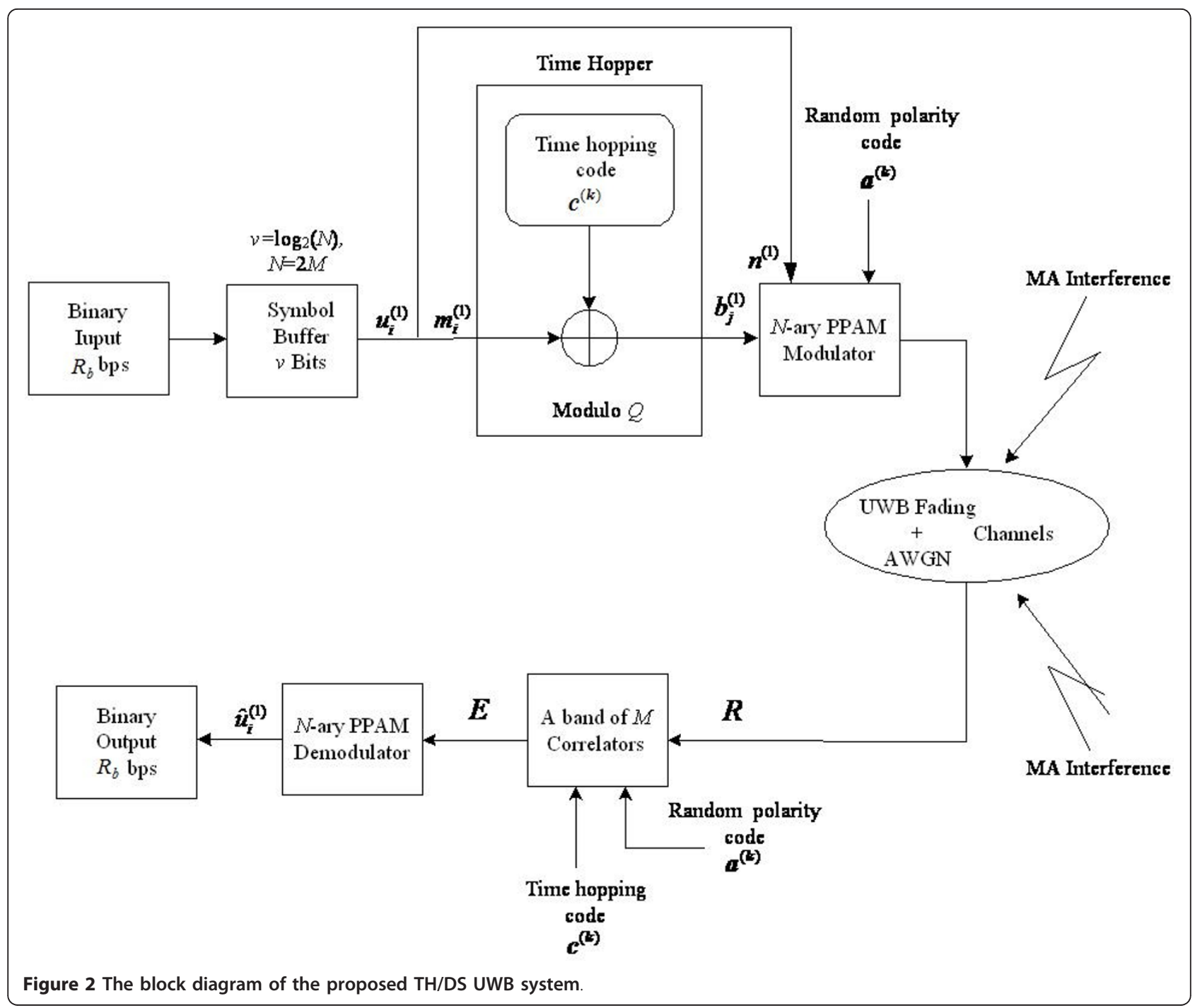

received signal is $r(t)=\sum_{k=1}^{K} A_{k} s_{r}^{(k)}\left(t-\tau_{k}\right)+n(t)$, where $K$ is the number of users, $A_{k}$ is the channel attenuation of the $k$ th signal $s_{r}^{(k)}(t), \tau_{k}$ is the propagation delay of the $k$ th signal, and $n(t)$ is the AWGN with zero mean and two-sided power spectral density; $N_{0} / 2$. Let $s_{r}^{(1)}(t)$ be the desired signal, and $u_{i}^{(1)}=\left(m_{i}^{(1)}, n_{i}^{(1)}\right)$ is the corresponding desired data symbol in the $i$ th symbol period. Assume that the desired signal is perfectly synchronized at the receiver. $\left(\tau_{1}=0\right)$ and $\left\{\tau_{k}\right\}_{k=2}^{K}$ are assumed to be uniformly distributed random variables over one symbol duration [4,13]. $s_{r}^{(k)}(t)=s^{(k)}(t) \otimes h^{(k)}(t)$, where $\otimes$ denotes convolution operation, $s^{(k)}(t)$ is the $k$ th user's signal of the DS, the conventional TH/DS, or the proposed TH/DS scheme described as (1), (2), or (4). $h^{(k)}(t)$ is the UWB channel model [22]. We also fix the required bandwidth $W=1 / T_{p}$ and the transmission bit rate $R_{b}=1 / T_{b}$ to have faire comparison of the performances among the systems. For the DS-UWB system, the bit interval is $T_{b}=T_{f}=N_{r} \times T_{p}$, and the processing gain is $T_{b} / T_{p}=N_{r}$ [13]. In cases of the conventional TH/DS system [3], we obtain

$$
\frac{T_{b}}{T_{p}}=\frac{\widehat{N}_{s} \times \widehat{N}_{c} \times \widehat{M}}{\log _{2} \hat{N}}
$$

in which the notations with $\widehat{(\cdot)}$ denote the system parameters used in the conventional TH/DS system. For the proposed TH/DS system, the symbol duration is

$$
\begin{aligned}
T_{s} & =N_{s} \times T_{f}=N_{s} \times Q \times T_{p} \\
& =\left(\log _{2} N\right) \times T_{b}
\end{aligned}
$$


and so the ratio of $T_{b}$ and $T_{p}$ is

$$
\frac{T_{b}}{T_{p}}=\frac{N_{s} \times Q}{\log _{2} N}
$$

\section{Performance analysis}

For the conventional TH/DS system using $N$-ary BPPM in AWGN channel, to detect the ith data symbol of the desired user $\widehat{u}_{i}^{(1)}$, the received signal is correlated with $\widehat{M}(=\widehat{N} / 2)$ orthogonal template waveforms to obtain $\widehat{M}$ decision statistics $\left\{r_{m}\right\}_{m=0}^{\widehat{M}-1}$ as follows [3]:

$$
r_{m}=\sum_{j=i \widehat{N}_{s}}^{(i+1) \widehat{N}_{s}-1} \int_{j T_{f}}^{(j+1) T_{f}} r(t) h_{m}\left(t-j T_{f}\right) d t=\left\{\begin{array}{cc}
S_{\text {conv }}+I_{\text {conv }}+n_{\text {conv }}, & m=m_{i}^{(1)} \\
I_{\text {conv }}+n_{\text {conv }} \quad & m \neq m_{i}^{(1)}
\end{array}\right.
$$
by

The template waveform of the $m$ th correlator is given

$$
h_{m}(t)=\sqrt{\frac{\widehat{N}_{s}}{E_{s}}} a_{j}^{(1)} p\left(t-c_{j}^{(1)} T_{c}-m \delta\right)
$$

where $S_{\text {conv }}=(-1)^{n_{i}^{(1)}} \widehat{N}_{s} A_{1} R(0)$ is the correlator output of the desired transmitted signal. $I_{\text {conv }}$ is the correlator output coming from other $K-1$ users' signals and is called MAI. The MAI can be described as $I_{\text {conv }}=\sum_{k=2}^{K} A_{k} I_{\text {conv }}^{(k)}$, where $I_{\text {conv }}^{(k)}$ is the MAI caused by the $k$ th user. $n_{\text {conv }}$ is a Gaussian random variable with zero mean and variance $\sigma_{n_{\text {conv }}}^{2}=N_{0} \widehat{N}_{s}^{2} R(0) / 2 E_{s}$. Based on the maximum likelihood decision rule for AWGN channel [5], the receiver of the desired user computes a bank of $M$ correlators' outputs, $\left\{r_{m}\right\}_{m=0}^{M-1}$ in (7), and then chooses the index corresponding to the largest absolute value of the correlator's output as the estimate of the message symbol $\widehat{m}_{i}^{(i)}$ :

$$
\widehat{m}^{(1)}=\underset{m \in\{0,1, \ldots N / 2-1\}}{\arg \max }\left|r_{m}\right|
$$

as well as

$$
\widehat{n}^{(1)}=\left\{\begin{array}{l}
1, r_{\widehat{m}^{(1)}}<0 \\
0, r_{\widehat{m}^{(1)}}>0
\end{array}\right.
$$

Consider the receiver structure of the proposed $\mathrm{TH} /$ DS system shown in Figure 3. The output of the $m$ th correlator in the $j$ th frame duration is

$$
e_{m j}=\int_{j T_{f}}^{(j+1) T_{f}} r(t) h_{m \oplus c_{j}^{(1)}}\left(t-j T_{f}\right) d t= \begin{cases}S_{j, \text { pro }}+I_{j, \text { pro }}+n_{j, \text { pro, }} & m=m_{i}^{(1)} \\ I_{j, \text { pro }}+n_{j, \text { pro, }} & m \neq m_{i}^{(1)}\end{cases}
$$

where $n_{j \text {,pro }}$ is i.i.d. Gaussian noise with zero mean and variance $\sigma_{n_{j, \text { pro }}}^{2}=N_{0} N_{s} R(0) / 2 E_{s}$. The template waveform of the $m$ th correlator is expressed as

$$
h_{m \oplus c_{j}^{(1)}}(t)=\sqrt{\frac{N_{s}}{E_{s}}} a_{j}^{(1)} p\left(t-\left(m \oplus c_{j}^{(1)}\right) \delta\right)
$$

where $S_{\text {conv }}=(-1)^{n_{i}^{(1)}} \widehat{N}_{s} A_{1} R(0)$ is the $m_{i}^{(1)}$ th correlator's output; $S_{j, \text { pro }}=(-1)^{n_{i}^{(1)}} A_{1} R(0)$ is the desired

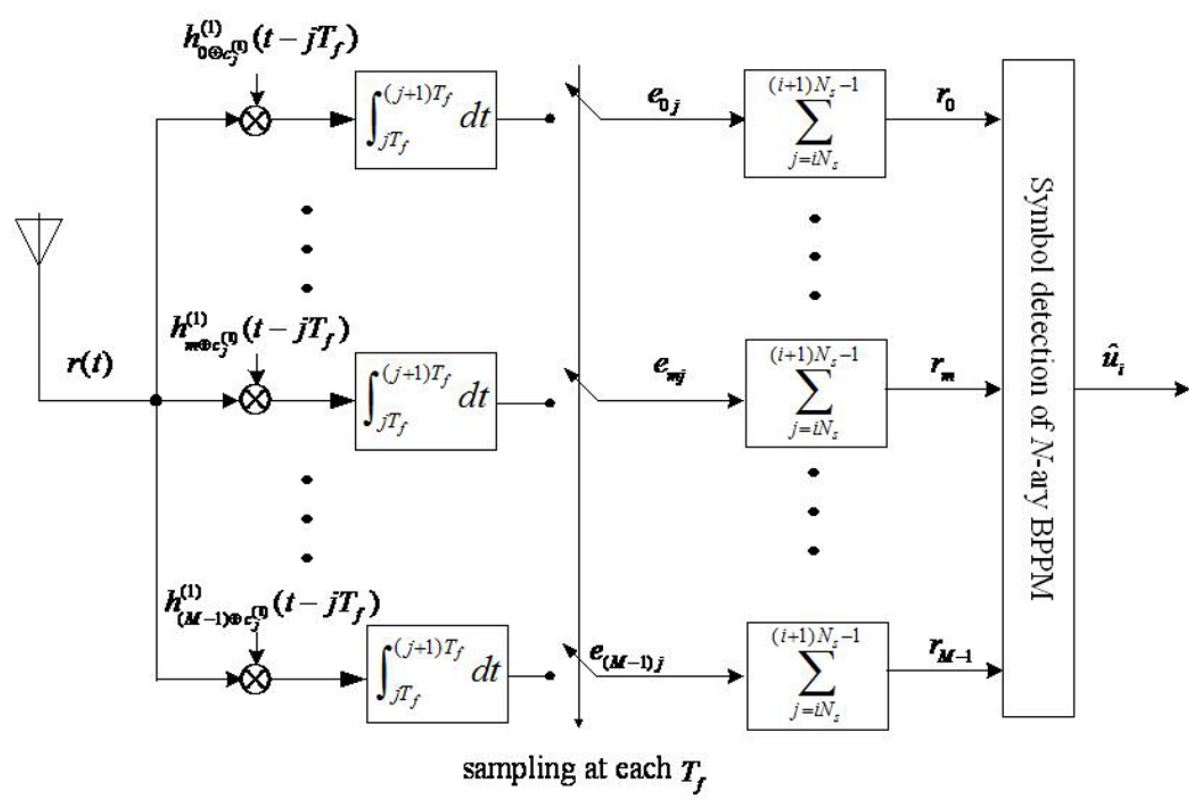

Figure 3 The receiver block diagram of the proposed TH/DS UWB system. 
component corresponding to the data symbol $m_{i}^{(1)}$, and $I_{j, \text { pro }}=\sum_{k=2}^{K} A_{k} I_{j, \text { pro }}^{(k)}$ where $I_{j \text {,pro }}^{(k)}$ is the MAI from the $k$ th user. Completing the combining process, the $m$ th decision variable can then be acquired as

$$
\begin{aligned}
r_{m} & =\sqrt{\frac{N_{s}}{E_{s}}} \sum_{j=i N_{s}}^{(i+1) N_{s}-1} a_{j}^{(1)} \int_{j T_{f}}^{(j+1) T_{f}} r(t) p\left(t-j T_{f}-\left(m \oplus c_{j}^{(1)}\right) \delta\right) d t \\
& =\left\{\begin{array}{c}
S_{\mathrm{pro}}+I_{\mathrm{pro}}+n_{\mathrm{pro}}, m=m_{i}^{(1)} \\
I_{\mathrm{pro}}+n_{\mathrm{pro}} \quad m \neq m_{i}^{(1)}
\end{array}\right.
\end{aligned}
$$

where $n_{\text {pro }}$ is Gaussian random noise with zero mean and variance $\quad \sigma_{n_{\mathrm{pro}}}^{2}=N_{0} N_{s}^{2} R(0) / 2 E_{s}$. $S_{\text {pro }}=(-1)^{n_{i}^{(1)}} A_{1} N_{s} R(0)$ is the desired component corresponding to the data symbol $m_{i}^{(1)}$, and $I_{\text {pro }}$ is the total MAI caused by the $K-1$ interfering users,

$$
I_{\text {pro }}=\sum_{j=i N_{s}}^{(i+1) N_{s}-1} I_{j, \text { pro }}=\sum_{j=i N_{s}}^{(i+1) N_{s}-1} \sum_{k=2}^{K} A_{k} I_{j, \text { pro }}^{(k)}
$$

Let $\tau_{k}=\alpha_{k} T_{f}+\Delta_{k}$ and $\Delta_{k}=\beta_{k} T_{p}+\gamma_{k}$, where $\alpha_{k}$ is the discrete uniformly distributed r.v. in $\left\{0,1, \ldots, N_{s}-1\right\} ; \beta_{k}$ is the discrete uniformly distributed r.v. in $\{0,1, \ldots, M$ $1\}$; and $\gamma_{k}$ is the continuous uniformly distributed r.v. in one pulse duration, i.e., $0 \leq \gamma_{k}<T_{p}$ [4]. Hence, we can obtain $I_{j, \text { pro }}^{(k)}$ as follows:

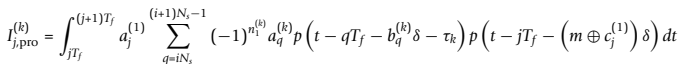

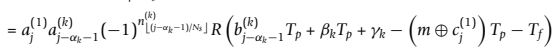

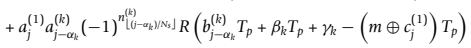

and can be rewritten as

$$
I_{j, \mathrm{pro}}^{(k)}=U_{j}^{(k)} \widehat{R}_{p}\left(\gamma_{k}\right) \widehat{\Gamma}_{j}\left(\Delta_{k}\right)+V_{j}^{(k)} R_{p}\left(\gamma_{k}\right) \Gamma_{j}\left(\Delta_{k}\right)
$$

$$
\text { where } \quad \widehat{R}_{p}\left(\gamma_{k}\right)=\int_{\gamma_{k}}^{T_{p}} p(t) p\left(t-\gamma_{k}\right) d t
$$
$U_{j}^{(k)} \cdot U_{j}^{(k)}$ and $V_{j}^{(k)}$ are the discrete uniformly distributed r.v.s in $\{-1,+1\}$ because the polarity codes $\mathbf{a}^{(k)}$ and the message symbol $n^{(k)}$ of the user $k$ are assumed to be random and equally likely. $\Gamma_{j}\left(\Delta_{k}\right)$ and $\widehat{\Gamma}_{j}\left(\Delta_{k}\right)$ can be expressed as follows:

$$
\begin{aligned}
\widehat{\Gamma}_{j}\left(\Delta_{k}\right) & =\left\{\begin{array}{l}
1 ; \text { if } 0 \leq k_{2}-k_{1}<T_{p} \text { or } 0 \leq k_{3}-k_{1}<T_{p} \\
0 ; \text { otherwise }
\end{array}\right. \\
\Gamma_{j}\left(\Delta_{k}\right) & =\left\{\begin{array}{l}
1 ; \text { if } 0 \leq k_{1}-k_{2}<T_{p} \text { or } 0 \leq k_{1}-k_{3}<T_{p} \\
0 ; \text { otherwise }
\end{array}\right.
\end{aligned}
$$

where $\quad k_{1}=\left(m \oplus c_{j}^{(1)}\right) T_{p}, k_{2}=\Delta_{k}+b_{j-\alpha_{k}}^{(k)} T_{p}, \quad$ and $k_{3}=\Delta_{k}+b_{j-\alpha_{k}-1}^{(k)} T_{p}-T_{f}$. The $i$ th data symbol of the $k$ th user $m_{i}^{(k)}$ is assumed to be an uniformly distributed r.v. in the range of $0 \leq m_{i}^{(k)} \leq M-1$ and each element $c_{j}^{(k)}$ of the random TH code utilized by the $k$ th user in the $j$ th frame period is assumed to be an uniformly distributed r.v. with $c_{j}^{(k)} \in G=\{0,1,2, \ldots, Q-1\}$. Therefore, $b_{j}^{(k)}=m_{i}^{(k)} \oplus c_{j}^{(k)}$ is an uniformly distributed r.v. with $b_{j}^{(k)} \in F$. The probability density function (PDF) of $I_{j, \mathrm{pro}}^{(k)}$ conditioned on $\gamma_{k}$ can be described as

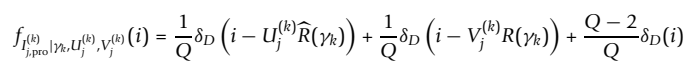

where $\delta_{\mathrm{D}}$ is the Dirac delta function. Therefore, the conditional CF of $I_{j, \text { pro }}^{(k)}$ can be obtained as obtained as

$$
\Phi_{I_{j, p r o}^{(k)} \mid \gamma_{k}}(\omega)=\frac{1}{Q} \cos \left(\omega R\left(\gamma_{k}\right)\right)+\frac{1}{Q} \cos \left(\omega \widehat{R}\left(\gamma_{k}\right)\right)+\frac{Q-2}{Q}(20)
$$

The interferences $I_{j, \text { pro }}^{(k)}$ are independent of each other because each element of the user's TH code $\mathbf{c}^{(k)}$ is randomly and independently selected from the set $G$. Hence,

$$
\Phi_{I_{\mathrm{pro}}^{(k)} \mid \gamma_{k}}(\omega)=\left[\frac{1}{Q} \cos \left(\omega R\left(\gamma_{k}\right)\right)+\frac{1}{Q} \cos \left(\omega \widehat{R}\left(\gamma_{k}\right)\right)+\frac{Q-2}{Q}\right]^{N_{s}}
$$

and we then obtain

$$
\begin{aligned}
& \Phi_{I_{\mathrm{pro}}^{(k)}}(\omega)=\frac{1}{T_{p}} \int_{0}^{T_{p}}\left[\frac{1}{Q} \cos \left(\omega R\left(\gamma_{k}\right)\right)+\frac{1}{Q} \cos \left(\omega \widehat{R}\left(\gamma_{k}\right)\right)+\frac{Q-2}{Q}\right]^{N_{s}} d_{\gamma_{k}} \\
& \Phi_{I_{\mathrm{pro}}}(\omega)=\prod_{k=2}^{K} \Phi_{I_{\mathrm{pro}}^{(k)}}\left(A_{k} \omega\right)
\end{aligned}
$$

It is worthy to note that the CF of the MAI component for each correlator's output of the proposed TH/ DS receiver is dixerent from that of the conventional TH/DS system which has been shown in [3].

\subsection{Symbol error rate}

Let the $i$ th data symbol of the desired user be $u_{i}^{(1)}=\left(m_{i}^{(1)}, n_{i}^{(1)}\right)=(0,0)$. According to our derived CF of the MAI component for the correlator's output of the proposed TH/DS receiver in (23), the average SER of 
the $\mathrm{N}$-ary biorthogonal modulation has been expressed and calculated as [5]:

$$
P_{e}=1-\int_{0}^{+\infty} P\left(\left|r_{1}\right| \leq \mu,\left|r_{2}\right| \leq \mu, \ldots,\left|r_{N / 2-1}\right| \leq \mu \mid \mu\right) f_{r_{0}}(\mu) d u
$$

The decision statistics of the combining correlators' outputs $\left\{r_{m}\right\}_{m=0}^{N / 2-1}$ are assumed to be independent [2,3,17-21]. Hence, the SER of the proposed system is

$$
\begin{aligned}
P_{e} & =1-\int_{0}^{+\infty}\left[P\left(\left|r_{1}\right| \leq u \mid u\right)\right]^{N / 2-1} f_{r_{0}}(u) d u \\
& =1-\int_{0}^{+\infty}\left[F_{r_{1}}(u)-F_{r_{1}}(-u)\right]^{N / 2-1} f_{r_{0}}(u) d u
\end{aligned}
$$

where $F_{r_{1}}(u)$ is the CDF of $r_{1}$. The first decision variable is $r_{0}=A_{1} N_{s} R(0)+I+n$, and the other $M-1$ decision variables are $\left\{r_{m}\right\}_{m=0}^{M-1}=I+n$. Therefore, (24) can be rewritten as

$$
P_{e}=1-\int_{0}^{+\infty}\left[F_{r_{1}}(u)-F_{r_{1}}(-u)\right]^{N / 2-1} f_{r_{1}}\left(u-A_{1} N_{s} R(0)\right) d u
$$

As the MAI and AWGN are assumed to be mutually independent, we obtain the CF of $r_{1}$ as $\Phi_{r_{1}}(\omega)=\Phi_{I}(\omega) \Phi_{n}(\omega)$, where the CF of the AWGN is $\Phi_{n}(\omega)=e^{-\sigma_{n_{\mathrm{pro}}}^{2} \omega^{2} / 2}$. Hence, the PDF of $r_{1}$ can be acquired as

$$
f_{r_{1}}(u)=\frac{1}{\pi} \int_{0}^{+\infty} \Phi_{r_{1}}(\omega) \cos (\omega u) d \omega
$$

Applying the relationship between the CF and CDF [12-14], we have

$$
F_{r_{1}}(u)=\frac{1}{2}+\frac{1}{\pi} \int_{0}^{+\infty} \Phi_{r_{1}}(\omega) \frac{\sin (\omega u)}{\omega} d \omega
$$

If all $M-1$ erroneous symbols are equally likely chosen, then the corresponding BER is $P_{b}=M \cdot P_{e} /[2 \cdot(M$ - 1)] [5].

\section{Simulation results}

The pulse width $T_{p}=0.7 \mathrm{~ns}$ is selected for the systems. Assuming that the system bandwidth and the data rate are fixed at $T_{b} / T_{p}=64$, the BER performances of the conventional and the proposed TH/DS systems are respectively illustrated in Figure 4 with $K$ $=16$, and in Figures 5 and 6 with $K=24$. Figure 4 shows the BER performances of the conventional and the proposed TH/DS systems using different modulation levels of BPPM with a fixed number of frames. In Figure 5, the SER performances of the conventional and the proposed TH/DS systems employing the same modulation level $\widehat{N}(N)=16$ are examined. It is

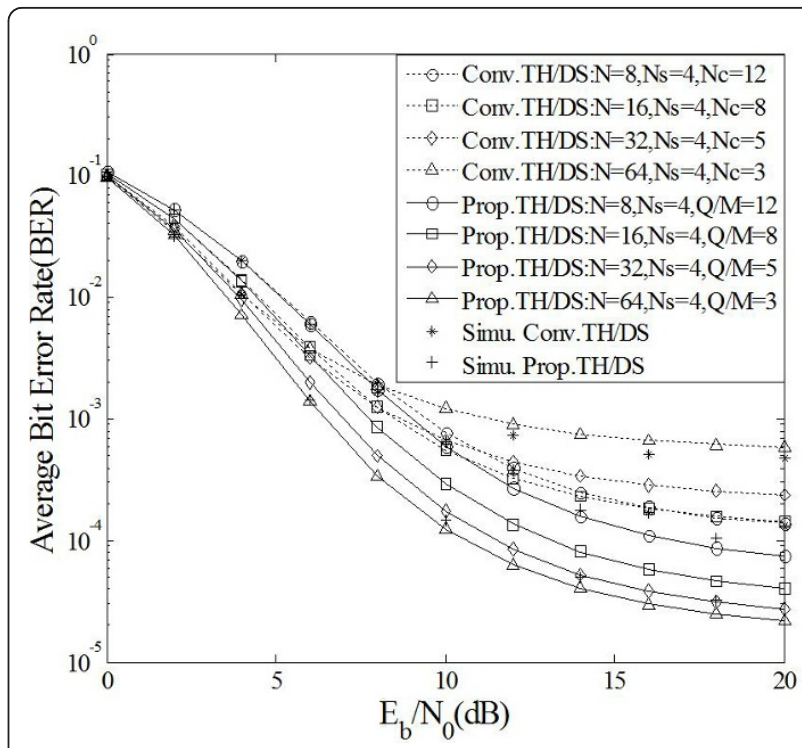

Figure 4 Average BERs versus $E_{b} / N_{o}$ of the conventional and the proposed TH/DS systems with various combinations of system parameters: $T_{b} / T_{p}=64, N_{s}=4$ and $K=16$.

observed that both TH/DS systems perform better as the number of frame is increased. Therefore, the conventional TH/DS scheme with no $\mathrm{TH}$ scenario $\left(\widehat{N}_{c}=1\right)$ and the proposed TH/DS system with $Q=M$ are with the best performance. In other words, for the conventional UWB systems employing BPPM signaling, DS-UWB system is more effective to combat MAI than the TH-UWB system [13]. On the other hands, although the number of available pulse slots $Q=M$ is

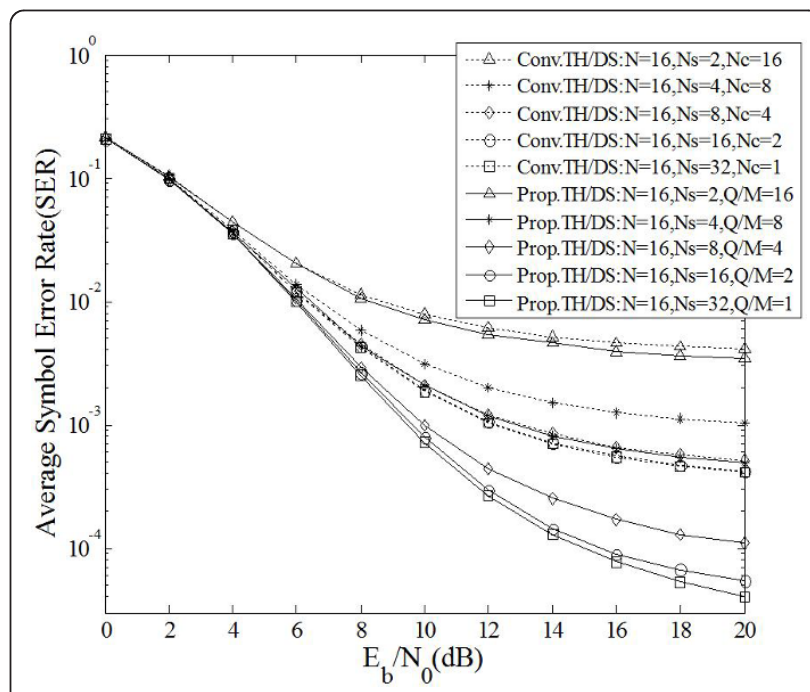

Figure 5 Average SER of the conventional and the proposed TH/DS UWB systems with different $\boldsymbol{N}_{s}$ and $\boldsymbol{N}_{c}: T_{b} / T_{p}=64, N=16$ and $K=24>$. 


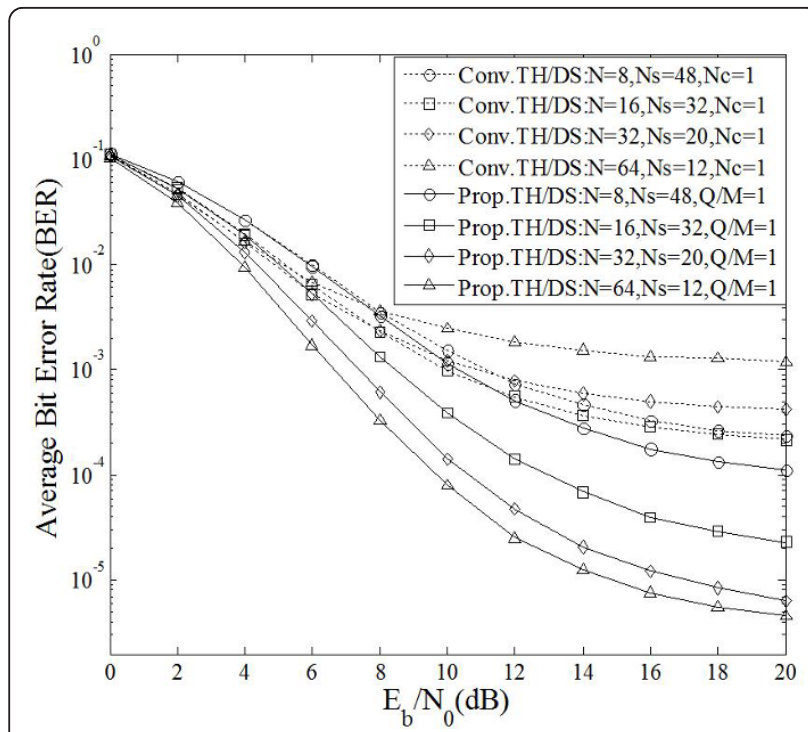

Figure 6 Performance comparisons of the conventional and the proposed TH/DS UWB systems: $T_{b} / T_{p}=64$ and $K=24$.

selected, which can provide the largest number of frames $N_{s}$ based on (6), the proposed TH/DS systems still utilize the whole frame duration to carry out the $\mathrm{TH}$ process and further reduce the impact of MAI. Figure 6 demonstrates the performance comparisons between the conventional and the proposed TH/DS systems employing several modulation levels accompanied with the largest number of frames used. From Figures 4,5 , and 6 , the performance of the conventional TH/DS system becomes worse with the modulation level $\widehat{N}>16$. However, applying larger modulation level in the proposed TH/DS system still provides lower average error probability. Even though smaller modulation levels employed in the conventional and the proposed TH/DS systems result in almost the same system complexities, the proposed $\mathrm{TH} / \mathrm{DS}$ system still outperforms the conventional TH/ DS scheme.

To achieve the best performance of the proposed TH/ DS system, the number of available pulse slots $Q=M$ is chosen in the following numerical results and simulations. The analytic SERs of the proposed system using different $N_{s}$ (the levels of time diversity) are presented in Figure 7. As expected, the performance of the proposed system becomes better as the number of frame is larger. It is noted that the performance gain is obtained at the cost of the reduction of the transmission (bit) rate. In Figure 8, the impact of the number of users on the performance of the proposed system is investigated. When we consider the fixed system throughput $K R_{b}$ in Figure 8 , it is shown that the proposed system applying larger $T_{b} / T_{p}(=64)$ and modulation level $(N=128)$ can

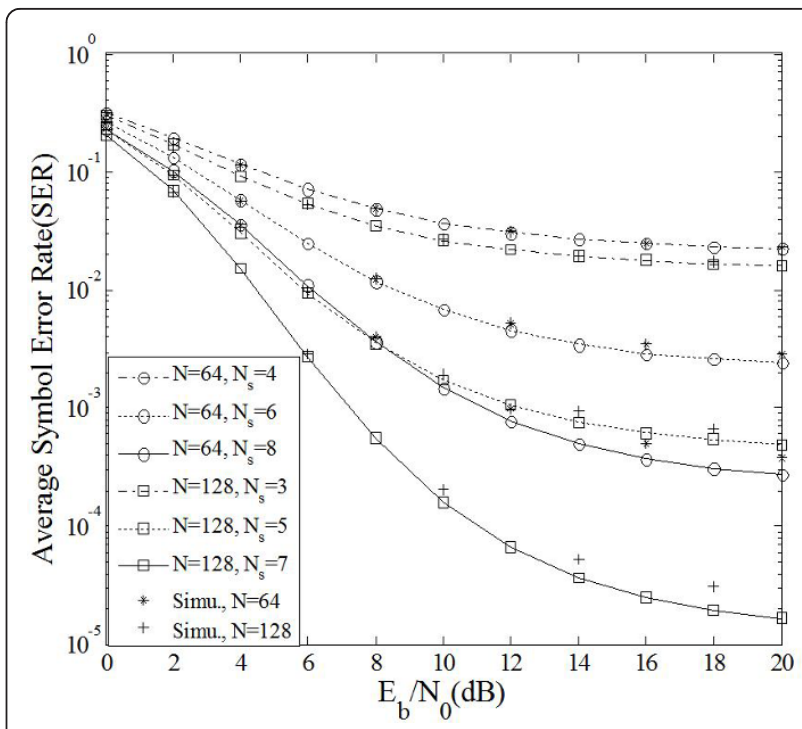

Figure 7 Average SER of the proposed TH/DS-UWB system for different number of frames. The number of modulation levels are $N=64 ; 128$ and the number of users is 24 .

provide better performance. From these aforementioned performance curves, the analytic results are consistent with the simulations.

Considering the cases of fixed ratios of $T_{b} / T_{p}=32,64$, and 128 (corresponding to bit rates $R_{b}=45 ; 22.5$ and $11.25 \mathrm{Mbps}$, respectively), the analytic SER performances of the proposed system with different number of modulation levels $N$ are depicted in Figure 9. According to (6), the number of frames (the level of time

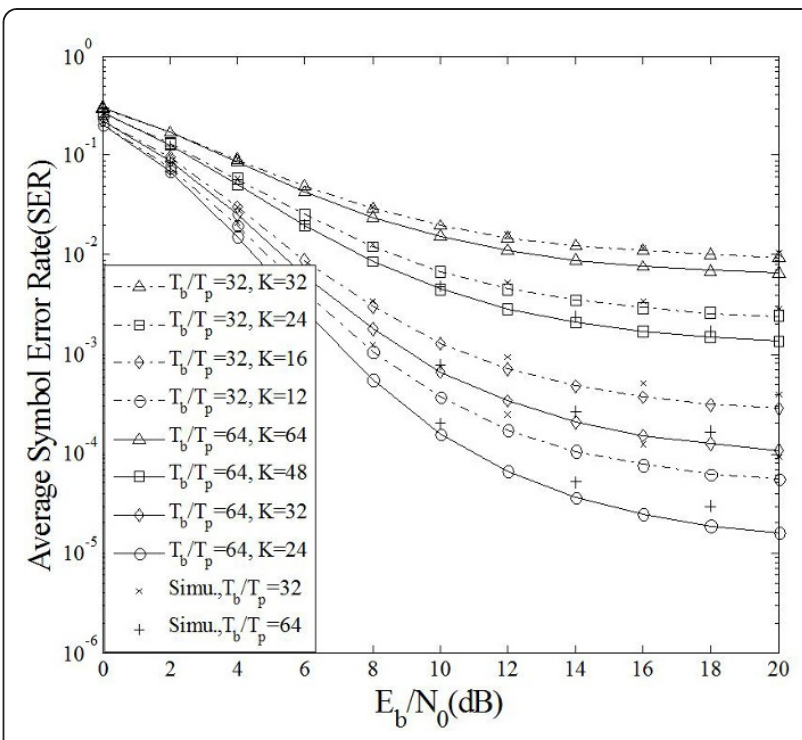

Figure 8 Average SER of the proposed TH/DS-UWB system for different number of asynchronous users. $N=64 ; N_{s}=6$ for $R_{b}=$ $45 \mathrm{Mbps}$ and $N=128 ; N_{s}=7$ for $R_{b}=22.5 \mathrm{Mbps}$. 


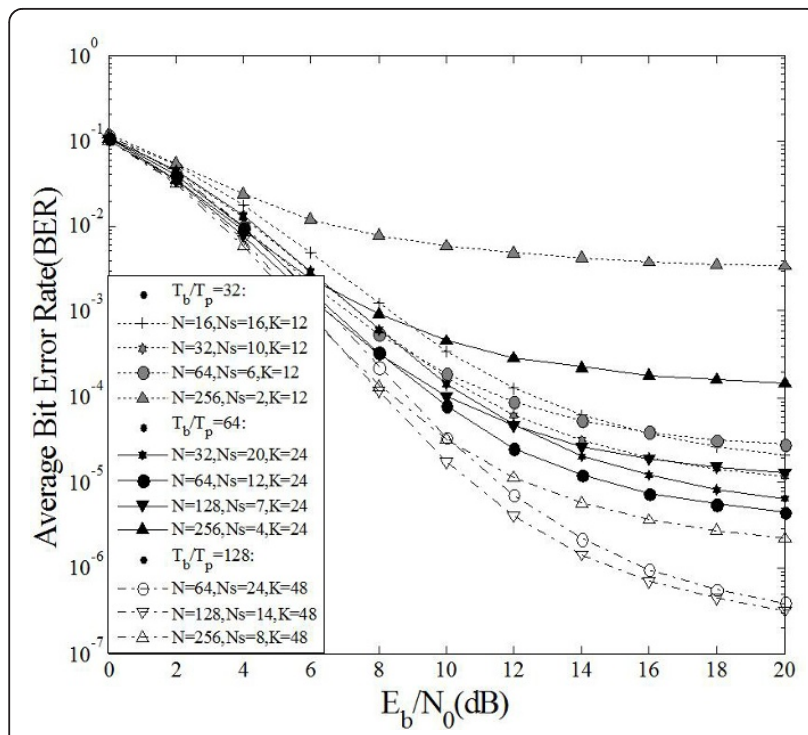

Figure 9 Average BER of the proposed TH/DS UWB system with various combinations of the system parameters under three bit rate $R_{b}=45 \mathrm{Mbps}, 22.5 \mathrm{Mbps}$ and $11.25 \mathrm{Mbps}$.

diversity) $N_{B}$ decreases as the number of modulation levels increases. To make fair performance comparison, the same system throughput, i.e., $K R_{b}=540 \mathrm{Mbps}$, is considered. Consequently, the numbers of users are $K=$ 12,24 , and 48 are utilized corresponding to $T_{b} / T_{p}=32$, 64 and 128, respectively. Observing the results in Figure 9 , for a specific $T_{b} / T_{p}$, there is an optimum $N$ that achieves minimum SER. The optimal $N$ is equal to $T_{b} /$ $T_{p}$. This result is similar to that of the frequency-hopping MA systems in [7]. In addition, the proposed system combined with larger $T_{b} / T_{p}(=N)$ can provide better SER performance.

In Figure 10, the BERs of the conventional TH/DSUWB, the proposed TH/DS-UWB, and the DS-UWB systems with the total number of users $K=16$ are examined. The cases of $T_{b} / T_{p}=32$ and 64 in [13] are considered. When the bit rate is $R_{b}=45 \mathrm{Mbps}$, the system parameters of the conventional TH/DS system are $\widehat{N}=16, \widehat{N}_{s}=16$ and $\widehat{N}_{c}=1$. On the other hand, the parameters of $N=16$ and $32\left(N_{s}=16\right.$ and 10) are selected in the proposed TH/DS system. In cases of $R_{b}$ $=22.5 \mathrm{Mbps}$, the optimal system parameters of the conventional TH/DS system which was shown in Figure 6 are $\widehat{N}=16, \widehat{N}_{s}=32$ and $\widehat{N}_{c}=1$. For the proposed TH/ DS system, the parameters of $N=16$ and $64\left(N_{s}=32\right.$ and 12) are chosen. This figure demonstrates that the performance of the proposed TH/DS system is better than that of the conventional TH/DS system even though the same system complexities (i.e., the same modulation levels) are considered. It is worthy to note that both the conventional and the proposed TH/DS-

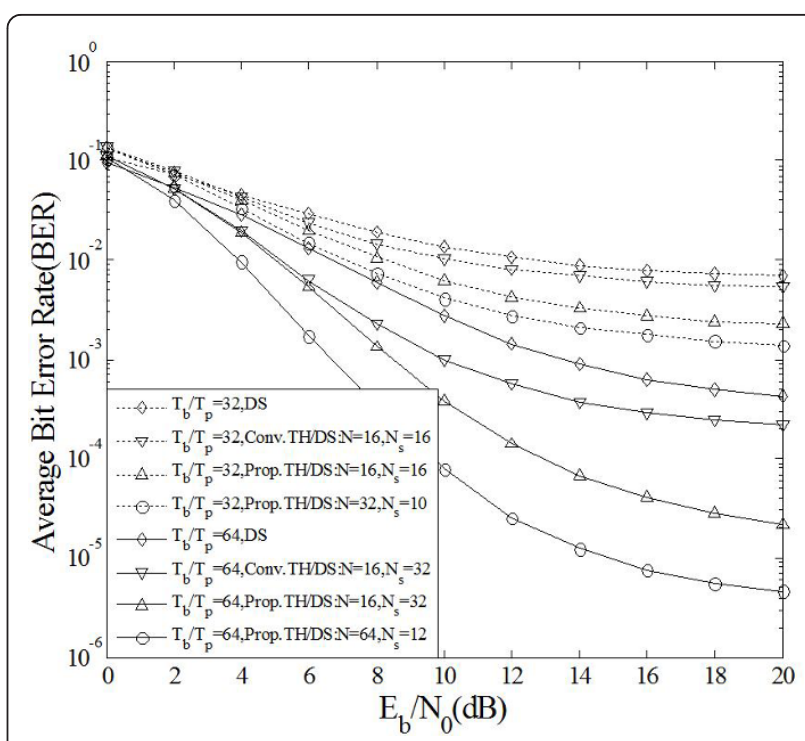

Figure 10 Performance comparisons of the conventional TH/ DS, the proposed TH/DS and the DS UWB systems with the bit rate $\boldsymbol{R}_{b}=\mathbf{4 5} \mathrm{Mbps}$ or $\mathbf{2 2 . 5} \mathrm{Mbps}$ : the number of users is 16 .

UWB systems employing non-binary BPPM are illustrated to outperform the DS-UWB system which was shown to provide the best system performance in all of the binary-modulated UWB systems [13].

Finally, the simulations of the conventional and the proposed TH/DS systems are conducted in the specific UWB multipath channel model, namely, CM3 fading channel. The partial RAKE (PRAKE) receiver that

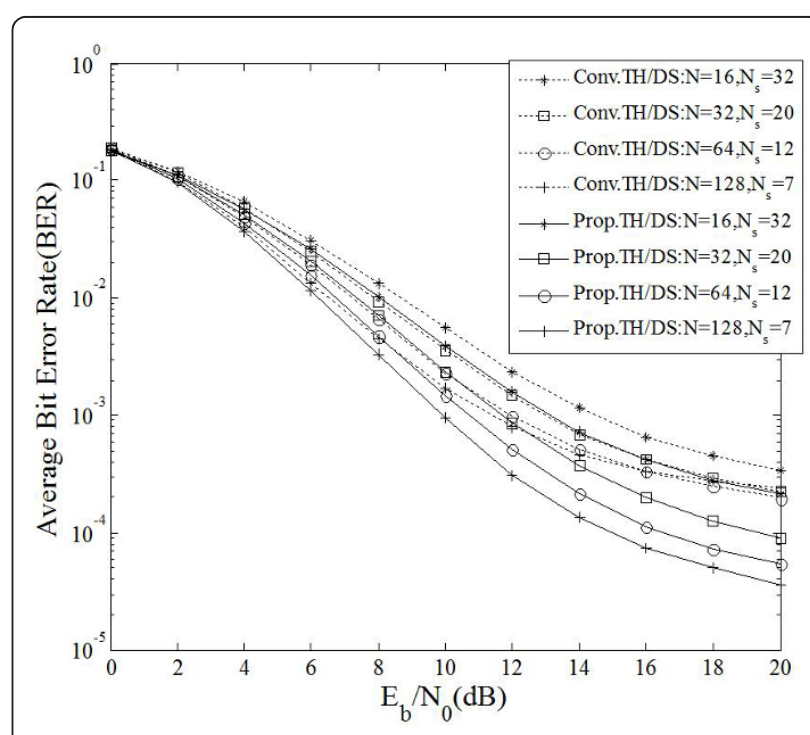

Figure 11 Performance comparisons of the conventional and the proposed TH/DS UWB systems with the partial Rake receiver in the CM3 UWB fading channel. The bit rate is 22.5 Mbps and the number of users is $K=16$. 
combines the first 20 resolvable multipaths is employed. Perfect channel estimation is assumed, and the maximum ratio combining is applied in the receiver. The BER performances of the examined TH/DS systems in CM3 fading channel are presented in Figure 11, in which all of the system parameters adopted are the same as those used in Figure 10. It is observed that the proposed TH/DS scheme performs better than the conventional TH/DS system.

\section{Conclusions}

A new TH/DS UWB system employing the whole frame to carry out TH and $N$-ary BPPM is proposed in this article. According to the derived CF expression of the MAI, a simple SER analysis of the proposed TH/DS system using $N$-ary BPPM is obtained in the presence of asynchronous MAI and AWGN. The proposed SER performance analysis is shown to be consistent with the simulations. From the simulation results, the proposed TH/DS system is shown to outperform both the conventional TH/DS system and the DS UWB systems.

The authors declare that they have no competing interests.

\section{Acknowledgements}

This study was supported in part by the National Science Council of Taiwan under Contract NSC 99-2221-E-150-042.

\section{Author details}

${ }^{1}$ Department of Aeronautical Engineering, National Formosa University, Yunlin, Taiwan ${ }^{2}$ Department of Electrical Engineering, National Chung-Hsing University, Taichung, Taiwan ${ }^{3}$ Department of Electronic Engineering, Chung Yuan Christian University, Chung Li, Taiwan

Received: 7 January 2011 Accepted: 31 October 2011

Published: 31 October 2011

\section{References}

1. MZ Win, RA Scholtz, Impulse radio: how it works. IEEE Commun Lett. 2(2), 36-38 (1998). doi:10.1109/4234.660796

2. H Zhang, TA Gulliver, Biorthogonal pulse position modulation for timehopping multiple access UWB communications. IEEE Trans Wirel Commun. 4(3), 1154-1162 (2005)

3. YS Shen, FB Ueng, An accurate performance analysis of hybrid TH/DS multiple access UWB system using N-ary biorthogonal PPM, in Proc IEEE VTC-Spring (2010)

4. MA Rahman, S Sasaki, J Zhou, H Kikuchi, Error analysis for a hybrid DS/TH impulse radio UWB multiple access system, in Proc IEEE ICC 2005, 220-224 (September 2005)

5. JG Proakis, Digital Communications (McGraw-Hill, New York, 2001)

6. DJ Goodman, PS Henry, VK Prabhu, Frequency-hopped multilevel FSK for mobile radio. Bell Syst Tech J. 59(7), 1257-1275 (1980)

7. UC Fiebig, The efficiency of FFH/CDMA systems in a mobile radio environment, in Proc IEEE ICC'94, 525-529 (1994)

8. MZ Win, RA Scholtz, Ultra-wide bandwidth time-hopping spread-spectrum impulse radio for wireless multiple-access communications. IEEE Trans Commun. 48(4), 679-689 (2000). doi:10.1109/26.843135

9. L Zhao, AM Haimovich, The capacity of an UWB multiple-access communications system, in Proc IEEE ICC 2002, 1964-1968 (May 2002)

10. J Zhang, TD Abhayapala, RA Kennedy, Performance of ultra-wideband correlator receiver using gaussian monocycles, in Proc IEEE ICC 2003, 2192-2196 (May 2003)
11. AR Forouzan, M Nasiri-Kenari, JA Salehi, Performance analysis of timehopping spread-spectrum multiple-access systems: uncoded and coded schemes. IEEE Trans Wirel Commun. 1(4), 671-681 (2002). doi:10.1109/ TWC.2002.804186

12. B Hu, NC Beaulieu, Accurate evaluation of multiple access performance in TH-PPM and TH-BPSK systems. IEEE Trans Commun. 52(10), 1758-1766 (2004). doi:10.1109/TCOMM.2004.836424

13. B Hu, NC Beaulieu, Accurate performance evaluation of time-hopping and direct-sequence UWB systems in multiuser interference. IEEE Trans Commun. 53(6), 1053-1062 (2005). doi:10.1109/TCOMM.2005.849792

14. S Niranjayan, A Nallanathan, B Kannan, Modeling of multiple access interference and BER derivation for TH and DS UWB multiple access systems. IEEE Trans Wirel Commun. 5(10), 2794-2804 (2006)

15. Y Dhibi, T Kaiser, On the impulsiveness of multiuser interferences in $\mathrm{TH}$ PPM-UWB systems. IEEE Trans Signal Process. 54(7), 2853-2857 (2006)

16. F Ramirez-Mireles, Performance of ultra wideband SSMA using time hopping and M-ary PPM. IEEE J Sel Areas Commun. 19(6), 1186-1196 (2001). doi:10.1109/49.926374

17. S Niranjayan, A Nallanathan, B Kannan, Modeling of multiple access interference and SER derivation for M-ary TH-PAM/PPM UWB systems, in Proc IEEE VTC 2005. 3, 2008-2012 (May 2005)

18. R Pasand, S Khalesehosseini, J Nielsen, A Sesay, Exact evaluation of M-ary TH-PPM UWB systems on AWGN channels for indoor multiple-access communications. IEE Proc Commun. 153(1), 83-92 (2006). doi:10.1049/ipcom:20045287

19. NV Kokkalis, PT Mathiopoulos, GK Karagiannidis, CS Koukourlis, Performance analysis of M-ary PPM TH-UWB systems in the presence of MUI and timing jitter. IEEE J Sel Areas Commun. 24(4), 822-828 (2006)

20. QF Zhou, FCM Lau, Analytical performance of M-ary time-hopping orthogonal PPM UWB systems under multiple access interference. IEEE Trans Commun. 56(11), 1780-1784 (2008)

21. H Zhang, W Li, TA Gulliver, Pulse position amplitude modulation for timehopping multiple-access UWB communications. IEEE Trans Commun. 53(8), 1269-1273 (2005). doi:10.1109/TCOMM.2005.852828

22. AF Molisch, JR Foerster, M Pendergrass, Channel models for UWB personal area networks. IEEE Wirel Commun Mag. 10(6), 14-21 (2003). doi:10.1109/ MWC.2003.1265848

doi:10.1186/1687-1499-2011-149

Cite this article as: Shen et al:: A new time-hopping/direct-sequence biorthogonal PPM UWB communication system. EURASIP Journal on Wireless Communications and Networking 2011 2011:149.

\section{Submit your manuscript to a SpringerOpen ${ }^{\circ}$ journal and benefit from:}

- Convenient online submission

- Rigorous peer review

- Immediate publication on acceptance

- Open access: articles freely available online

- High visibility within the field

- Retaining the copyright to your article

Submit your next manuscript at $>$ springeropen.com 